\title{
The lady who went twenty years back in her life - a case of acute bilateral thalamic infarction due to artery of percheron involvement
}

\author{
de Silva $\mathrm{CM}^{1}$, Fonseka $\mathrm{CL}^{1}$, Singhapura SDAL ${ }^{1}$, Kularatne $O \mathrm{AR}^{1}$, Jayasekara JMS ${ }^{1}$, Rajapaksha $\mathrm{H}^{2}$, \\ Dissanayake AS', Pathirana KD'
}

Journal of the Ceylon College of Physicians, 2017, 48, 85-87

\section{Introduction}

The thalamic and midbrain region of the brain is supplied by a complex arrangement of blood vessels fed by a large number of arteries ${ }^{1,2}$. Although variations exist, the perforating branches from the posterior cerebral artery and the posterior communicating artery provides the main arterial supply to this area ${ }^{3}$. The artery of Percheron (AOP) is a rare variant of this blood supply, with a frequency of occurence of $4-10 \%$ of all thalamic strokes and $0.2-2 \%$ of all ischaemic strokes ${ }^{4}$. The AOP is a single trunk which arises from the first segment of the posterior cerebral artery and supplies blood to the thalamus bilaterally ${ }^{5}$. Occlusion of this common arterial trunk leads to typical mirror-like symmetric bilateral thalamic infarctions which could be considered pathognomonic of occlusion of the artery of Percheron.

Various clinical patterns of presentation could occur including behavioral amnesic impairment, mental status disturbance, dysarthria, ocular movement disorders or gaze palsy and motor or cerebellar deficits. Behavioral amnesic impairment includes confusion, agitation, apathy, disinhibition, hyperphagia, pseudobulbar affect or any type of amnesia ${ }^{6,7}$. In patients with amnestic impairment, neurological examination reveals no clinically demonstrable focal neurological deficit in acute setting, making this diagnosis easily missed.

We describe a patient who presented with acute memory impairment who was subsequently diagnosed of having a bilateral thalamic infaction.

\footnotetext{
${ }_{1}^{1}$ University Medical Unit, Teaching Hospital, Galle, Sri Lanka.

${ }^{2}$ University Psychiatry Unit, Teaching Hospital, Galle, Sri Lanka.

Corresponding author: Fonseka $\mathrm{CL}$

E-mail: fonseka.lakmal@gmail.com
}

\section{Case report}

A 71-year-old woman with no significant past medical history, last seen awake 10 hours earlier, was found unresponsive on her bed by her daughter in the morning. Although repeated attempts were made to wake her up, she was drowsy and did not show appropriate response to voice or movement. After hospital admission, she was drowsy with a Glasgow Coma Scale of 6 with a pulse rate of 78 beats $/ \mathrm{min}$, respiratory rate 17 breaths/min, blood pressure 120/ $62 \mathrm{mmHg}$ with a peripheral oxygen saturation of $98 \%$ on room air. Pupillary sizes and light reflexes were normal bilaterally and there was no gaze palsy. The patient was afebrile and no meningeal signs were present. There was no apparent limb weakness and plantar reflexes were flexor in both sides. The following laboratory tests were all unremarkable including complete blood count, glucose, electrolytes, coagulation tests, liver enzymes, creatinine, ammonia, arterial blood gas values. Toxicology screening did not reveal excessive ethanol, benzodiazepine and opioid levels in blood. The initial CT performed 60 minutes after finding the patient showed no acute intracerebral hemorrhage or early signs of ischemia. Patient was hydrated and observed in ward and after 6 hrs of hospital admission, patient gradually regained consciousness and she became fully conscious and rational within the next hour. Neurological examination did not reveal any focal deficits.

On close communication with the patient, the relatives noticed that she could only remember incidents which has happened twenty years ago. She remembered that her daughter was newly married and was living in her home town. Although she could face recognize all relatives and communicate with them, she could only relate them with the memories twenty years go. She also mentioned about the president of the country who was there before twenty years. Detailed cognitive assessment revealed that the patient is having a memory loss over the last twenty-year duration and inability to retain the recent events in her memory. She had a MMSE score of $18 / 30$ with 
predominent impairment of recent memory. She has also forgotten the recent activities that she has been doing early within the day. She has been a very religious person, worshipping daily and she is a person who prefers to stay at her own home most of the day. However, after the incident, she did not practice her daily worshipping and neither she was interested to go to her own house. She remained friendly with her relatives during conversation, but frequently realized that she cannot remember the early events of the day, and she did not confabulate. Detailed cognitive and memory assessment revealed that she is having anterograde and retrograde amnesia.

She underwent MRI brain, which reveled bilateral symmetrical thalamic infarctions (Figure 1). She was commenced on antiplatelet and statin therapy and was screened for diabetes and dyslipidemia. 2D echocardiography and carotid duplex did not reveal a source of thrombo-embolism. Her memory impairment persisted throughout hospital stay and up to 6-month follow-up visit. She was followed up by a neurologist and a psychologist. On re-examination she was found to have ongoing retrograde and anterograde amnesia. Repetitive orientation and counselling was performed for the patient and family members.

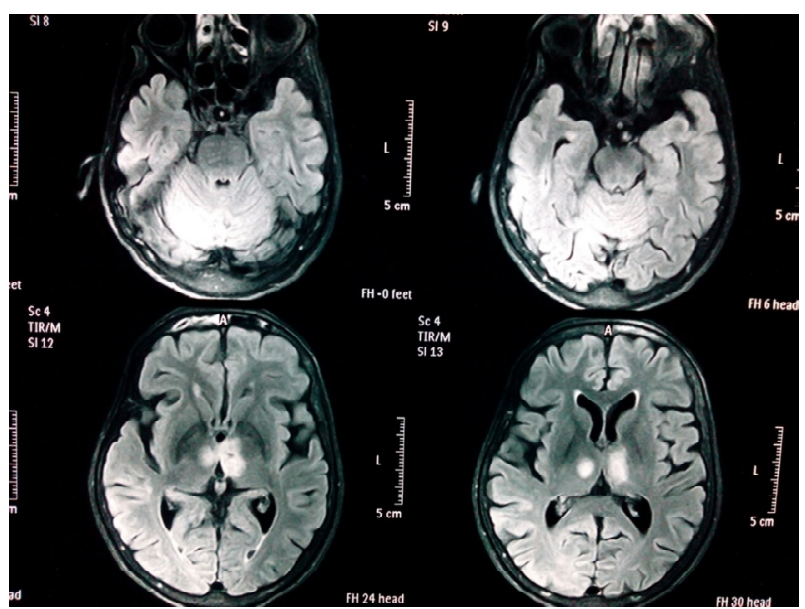

Figure 1. Magnetic resonance imaging showing bilateral thalamic infarction.

\section{Discussion}

Artery of Percheron occlusion resulting in bilateral paramedian thalamic infarction is a rare cause of amnesia or altered mental status. Our case highlights the importance of considering AOP territory ischemic stroke as a differential diagnosis of acute onset amnesia in the elderly, even though initial non-contrast CT was normal. In the acute setting, presenting symptoms could mimic conditions like encephalitis, non-convulsive status epilepticus and metabolic/toxic encephalopathy. A careful and detailed evaluation to exclude the above could point towards this rare entity. The acute, bizarre nature of presentation could distract clinicians from suspecting this condition, which can delay time sensitive treatment for an ischemic stroke syndrome. We found one case of successful intraarterial thrombolysis with an AOP infarction in literature?. Other than thrombosis of AOP, embolic occlusion of distal basilar artery resulting in 'top of the basilar artery syndrome' could mimic AOP infarction ${ }^{8}$ both clinically and radiologically, giving rise to bilateral thalamic infarctions.

Predominant anterograde and retrograde amnesia is rarely reported with bilateral thalamic infarction. Although, it usually occurs associated with several other focal neurological deficits, our patient had relatively isolated anterograde and retrograde amnesia with minor cognitive impairment at the beginning. There was also a minor change in her personality traits.

AOP infarction in the long-term has a generally favourable clinical outcome, except in those with associated midbrain infarction ${ }^{10}$. However, the management problems arise in managing the shortterm memory impairment. Patient necessitated repeated orientation and education about her current state as she forgot the instructions given to her about her illness within few hours or days. Several methods of documentation used in notepads and booklets were improvised to reduce forgetfulness. As she retained ability to read, write and understand, these methods were used to minimize forgetfulness.

\section{Conclusion}

Bilateral thalamic infarction due to artery of Percheron involvement is a rare stroke syndrome, and presentation with isolated retrograde amnesia with anterograde amnesia is even rarer. Presentation with isolated amnesia with minor cognitive impairment could delay the diagnosis of a stroke.

\section{References}

1. Krampla W, Schmidbauer B, Hruby W. Ischaemic stroke of the artery of Percheron. Eur Radiol 2008; 18: 192-4.

2. Matheus MG, Castillo M. Imaging of acute bilateral paramedian thalamic and mesencephalic infarcts. $A m \mathrm{~J}$ Neuroradiol 2003; 24: 2005-8.

3. Godani M, Auci A, Torri T, Jensen S, Sette DM. Coma with vertical gazepalsy. Relevance of angio-CT in acute percheron artery syndrome. Case Rep Neurol 2010; 2: 74-9. 
4. Urska Lamot, Ivana Ribaric, Katarina Surlan Popovic. Artery of Percheron infarction: review of literature with a case report. Radiol Oncol. 2015 ; 49(2): 141-6.

5. Arauz A, Patiño-Rodríguez $\mathrm{H}$, Vargas-González JC, Arguelles-Morales N, Silos H, Ruiz-Franco A, Ochoa MA. Clinical Spectrum of Artery of Percheron Infarct: ClinicalRadiological Correlations. J Stroke Cerebrovasc Dis. 2014; 23(5): 1083-8.

6. Bogousslavsky J, Regli F, Uske A. "Thalamic infarcts: clinical syndromes, etiology, and prognosis". Neurology 1988; 38: 837-48.
7. de la Cruz-Cosme C, Marquez-Martinez M, Aguilar-Cuevas $\mathrm{R}$, et al. Percheron artery syndrome: variability in presentation and differential diagnosis. Rev Neurol 2011; 53: $193-200$.

8. Kostanian V, Cramer SC. Artery of Percheron thrombolysis. Am J Neuroradiol 2007; 28: 870-71.

9. Rodriguez EG, Lee JA. Bilateral thalamic infarcts due to occlusion of the artery of Percheron and discussion of the differential diagnosis of bilateral thalamic lesions. J Radiol Case Rep 2013; 7: 7-14. 